\title{
COMMENT
}

\section{Rethinking informed consent in pediatric research: a time for regulatory policy change?}

\author{
Jean L. Raphael ${ }^{1}$ and Shale L. Wong ${ }^{2}$ on behalf of Pediatric Policy Council \\ Pediatric Research (2018) 84:477-478; https://doi.org/10.1038/s41390-018-0151-4
}

As the scope and volume of pediatric research continue to expand, protection of children is essential in order to advance medical knowledge and provide societal benefit. Informed consent represents a foundational element in the conduct of pediatric research. ${ }^{1,2}$ Our rules that guide informed consent stem from the Nuremberg trials during which 10 basic tenets were defined that provide informational parameters for consent to participate in an experimental study. ${ }^{3}$ These rules make no mention of children or specification around consenting on behalf of children. For consent to qualify as informed, the subject must receive and understand requisite information that will guide them in making an autonomous and voluntary decision. ${ }^{1}$ Obtaining consent requires informing the subject of his or her rights, the aims of the study, research procedures, potential risks and benefits of participation, expected duration of the study, and the extent of confidentiality. Appropriate transfer of information serves to provide both legal and ethical protections to both the child and researcher. ${ }^{2}$ The American Academy of Pediatrics (AAP) recommends that informed consent be conducted and legal authorization be obtained before any research is undertaken in children. ${ }^{4}$ Informed consent in pediatric research represents a unique challenge in that it involves adults and children. Depending on the age of the child, what is needed varies between parent permission and parent permission with child assent. Despite evidence that long informed consent forms (ICFs) can negatively impact potential participants' understanding of research, ${ }^{5}$ the length of ICFs has increased over time..$^{5-7}$ In this issue, Murray et al. examine whether a shortened and simplified ICF, accompanied by an appendix, improves participant understanding of a hypothetical neonatal study compared to a conventional ICF. ${ }^{8}$ The authors hypothesized that a shortened ICF with an attached appendix would be equivalent to a conventional ICF in terms of the understanding of key elements of informed consent. Furthermore, they hypothesized that parents would find the shortened ICF easier to read and understand. The authors did not explore specifically whether the shorter form meets the preferred needs of the patient and parent. In this study, the authors show that significantly more parents in the shortened ICF group found their form "short and to the point." They also stated the shortened ICF did not provide enough information (although we do not know what information was specifically lacking). There were no significant differences between groups measuring understanding of central study components. These findings demonstrate the potential of a shortened ICF with appendix to simplify the process of informed consent. More broadly, the findings from this study highlight some of the core controversies in the debate on how to simplify ICFs, preserve understanding of key concepts, and meet participant expectations that adequate information has been provided. We are challenged with an ethical question: is simplification warranted when personal specification is sacrificed? The challenge of balancing these aims is further compounded when informed consent requires both parent and child. In this commentary, we focus on the processes of informed consent in pediatrics and the need to study regulatory policy changes to optimize ICFs in pediatric research.

In an ICF, the research plan of a given study must be clearly explained with all the details, including procedures, potential benefits, and risks. ${ }^{2}$ The ability of parents and children to comprehend written medical information has been demonstrated to be variable. ${ }^{2}$ Estimates show that $14-22 \%$ of U.S. adults are functionally illiterate and even carefully written information can be difficult to understand. ${ }^{9}$ In a study on health literacy in the emergency care setting, $25 \%$ of adults had marginal or inadequate health literacy. ${ }^{10}$ These findings highlight the concerns influencing how much and what types of information should be included in ICFs. When parent understanding is potentially compromised, we should expect that children may also have difficulties in comprehending ICFs. As part of regulatory policy, in addition to parental agreement to enroll their child into a study, the child (given a certain age) is also expected to express agreement, termed as "assent." The AAP acknowledges children with an intellectual age of 7 years of age or older as capable of providing assent. ${ }^{11}$ However, a number of studies demonstrate that children may be limited in their comprehension of research and therefore overall capacity to consent to research. In one study of assent in the setting of a Hepatitis B vaccine trial, only $56 \%$ of children ages $12-17$ years of age demonstrated absolute comprehension. ${ }^{12}$ In another study on nutrition with age appropriate information that was explained orally coupled with written documents, none of the children under 9 years could describe the purpose of the study. ${ }^{13}$ In a study by Hein et al., investigators tested a competence assessment tool for children among subjects $6-17$ years of age. ${ }^{14}$ The tool found $37.9 \%$ of children incompetent to give consent, with competence in children younger than 9.6 years unlikely. Putting these findings in the larger context of studies on adult health literacy, it is clear that new familycentered approaches are needed to improve the informed consent process in pediatric research.

As researchers, ethicists, institutional review boards (IRBs), and patient advocacy groups debate the theoretical merits of modifying ICFs, the evidence base for shortening ICFs is slowly emerging. Shorter ICFs have been shown to be as effective or better than long ICFs at conveying relevant information and enhancing participants' comprehension. ${ }^{5,15-17}$ A recent study by Corneli et al. identified strategies to shorten ICFs in research. ${ }^{18}$ The 
investigators engaged a diverse set of stakeholders (participants in a research trial, scientists and staff, community representatives, research oversight staff, IRB members) to identify concerns with ICFs, determine strategies to improve ICFs, and anticipate challenges to implementing such strategies. Three overarching strategies were identified to reduce the overall length of ICFs: (1) group study procedures by frequency instead of study visit; (2) provide reference information about specific study procedures in appendix; and (3) list duplicative side effects once rather than listing all side effects for each drug. Barriers listed for implementation of the three strategies included researcher refusal to make changes, rigidness of IRBs to accept new approaches, reviewer expectations for research protocols, and protection from liability. Despite the existence of this growing literature supporting shorter ICFs and the strategies to apply, implementation of modifications to shorten ICFs has not been widely adopted.

The evidence collected to date on ICF length points to research and policy agendas for optimizing ICFs. ${ }^{18}$ Future studies could compare ICFs using the three strategies outlined above to traditional ICFs in a randomized controlled trial embedded within actual research studies. It would be valuable to study other innovative approaches incorporating shortened ICFs including use of different forms of multimedia for ICF presentation and group counseling where parents have the opportunity to hear research information, review research information sheets (i.e., short explanations of a research study), and interact with the investigator and other parents to enhance understanding. ${ }^{19-21}$ Potential outcomes would include participant understanding of the study, satisfaction with the informed consent process, and time saved by using a shorter process. ${ }^{18}$

Additional rigorous research coupled with advocacy efforts to influence regulatory policy change in how ICFs are written could promote uptake. ${ }^{2}$ Patient-centered engagement of research participants and family advocacy groups will elucidate their perceptions and comfort with shorter ICFs. This exploration will help us understand whether a shortened form could be devised to include preferred elements to meet both required relevant components for protection and those that support the patient's immediate interests, addressing the ethical conundrum of a comprehensive ICF that is beyond comprehension. Researchers and study staff who develop ICFs must be educated about evidence-based strategies to reduce ICF length. There is also a need to make IRB members and regulatory representatives aware of current approaches to shortened ICFs as part of continuing education. With engagement of diverse stakeholders, it is possible to create an informed consent process that preserves the ethical and legal principles of human subjects research, maximizes participant understanding, and promotes a new level of patientcentered approaches for pediatric research.

\section{ADDITIONAL INFORMATION}

Competing interests: The authors declare no competing interests.

Publisher's note: Springer Nature remains neutral with regard to jurisdictional claims in published maps and institutional affiliations.

\section{REFERENCES}

1. Pandiya, A. Readability and comprehensibility of informed consent forms for clinical trials. Perspect. Clin. Res. 1, 98-100 (2010).

2. Leibson, T. \& Koren, G. Informed consent in pediatric research. Paediatr. Drugs 17, 5-11 (2015).

3. Trials of War Criminals before the Nuremberg Military Tribunals under Control Council Law No. 10, Vol. 2, pp. 181-182. Washington, D.C.: U.S. Government Printing Office, 1949.

4. Katz A. L., Webb S. A., Committee On B. Informed Consent in Decision-Making in Pediatric Practice. Pediatrics. 2016;138.

5. Beardsley, E., Jefford, M. \& Mileshkin, L. Longer consent forms for clinical trials compromise patient understanding: so why are they lengthening? J. Clin. Oncol. 25, e13-e14 (2007).

6. Albala, I., Doyle, M. \& Appelbaum, P. S. The evolution of consent forms for research: a quarter century of changes. IRB 32, 7-11 (2010).

7. Berger, O., Gronberg, B. H., Sand, K., Kaasa, S. \& Loge, J. H. The length of consent documents in oncological trials is doubled in twenty years. Ann. Oncol. 20, 379-385 (2009).

8. Murray P. D., Bierer B. E., Hirschfeld S., Klein A. K., Davis J. M. Assessment of a shortened informed consent form for pediatric research: a pilot study. Pediatric Res. (2018).

9. Kutner, M., Greenberg, E., Jin, Y., and Paulsen, C. The Health Literacy of America's Adults: Results From the 2003 National Assessment of Adult Literacy (NCES 2006-483). U.S. Department of Education. Washington, DC: National Center for Education Statistics, 2006.

10. Carpenter, C. R. et al. Feasibility and diagnostic accuracy of brief health literacy and numeracy screening instruments in an urban emergency department. Academic emergency medicine: official journal of the Society forAcademic. Emerg. Med. 21, 137-146 (2014).

11. Shaddy, R. E. \& Denne, S. C., Committee on D, Committee on Pediatric R. Clinical report-guidelines for the ethical conduct of studies to evaluate drugs in pediatric populations. Pediatrics 125, 850-860 (2010).

12. Lee, S. et al. Comprehension of a simplified assent form in a vaccine trial for adolescents. J. Med. Ethics 39, 410-412 (2013).

13. Ondrusek, N., Abramovitch, R., Pencharz, P. \& Koren, G. Empirical examination of the ability of children to consent to clinical research. J. Med. Ethics 24, 158-165 (1998).

14. Hein, I. M. et al. Accuracy of the MacArthur competence assessment tool for clinical research (MacCAT-CR) for measuring children's competence to consent to clinical research. JAMA Pediatr. 168, 1147-1153 (2014).

15. Dresden, G. M. \& Levitt, M. A. Modifying a standard industry clinical trial consent form improves patient information retention as part of the informed consent process. Academic emergency medicine: official journal of the Society for. Acad. Emerg. Med. 8, 246-252 (2001).

16. Enama, M. E. et al. Randomization to standard and concise informed consent forms: development of evidence-based consent practices. Contemp. Clin. Trials 33, 895-902 (2012).

17. Matsui, K., Lie, R. K., Turin, T. C. \& Kita, Y. A randomized controlled trial of short and standard-length consent forms for a genetic cohort study: is longer better. J. Epidemiol. 22, 308-316 (2012)

18. Corneli, A. et al. Evidence-based strategies for shortening informed consent forms in clinical research. J. Empir. Res. Human. Res. Ethics12, 14-25 (2017).

19. Tait, A. R., Voepel-Lewis, T., McGonegal, M. \& Levine, R. Evaluation of a prototype interactive consent program for pediatric clinical trials: a pilot study. J. Am. Med. Inform. Assoc. 19, e43-e45 (2012).

20. O'Lonergan, T. A. \& Forster-Harwood, J. E. Novel approach to parental permission and child assent for research: improving comprehension. Pediatrics 127, 917-924 (2011).

21. Gutierrez-Castrellon, P. et al. Diarrhea in preschool children and Lactobacillus reuteri: a randomized controlled trial. Pediatrics 133, e904-e909 (2014). 